\title{
Devolopmental and growth temperature regulation of omega-3 fatty acid desaturase genes in safflower (Carthamus tinctorius L.)
}

\author{
L.-L. Guan' ${ }^{1}$ W. Wu ${ }^{2}$, B. Hu${ }^{2}$, D. $\mathrm{Li}^{2}$, J.-W. Chen ${ }^{2}$, K. Hou ${ }^{2}$ and L. Wang ${ }^{1}$ \\ ${ }^{1}$ Tropical Crops Genetic Resources Institute, \\ Chinese Academy of Tropical Agricultural Sciences / \\ Key Laboratory of Crop Gene Resources and Germplasm Enhancement in \\ Southern China, Ministry of Agriculture, China \\ ${ }^{2}$ Agronomy College, Sichuan Agricutural University, Cheng Du, China \\ Corresponding author: W. Wu / L.-L. Guan \\ E-mail: ewuwei@gmail.com / gllgirl123@163.com
}

Genet. Mol. Res. 13 (3): 6623-6637 (2014)

Received April 4, 2013

Accepted September 4, 2013

Published August 28, 2014

DOI http://dx.doi.org/10.4238/2014.August.28.7

\begin{abstract}
Three $\omega-3$ fatty acid desaturase genes (CtFAD3, CtFAD7, and CtFAD8) were isolated from safflower (Carthamus tinctorius L.). Transcript analysis showed that the highest transcript levels were detected for $C t F A D 3$ and the low transcript levels were detected for CtFAD7 and CtFAD8 in flowers. This result indicates that $C t F A D 3$ enzyme activity is important for fatty acid desaturation in flowers. The low transcript level of CtFAD 3 in developing seeds was consistent with the recorded high level of linoleic acid (18:2) and lack of linolenic acid (18:3) in safflower seed oil. At low temperatures, the induced transcription levels of $\omega-3$ fatty acid desaturase genes in the stems and petioles were consistent with increased polyunsaturated fatty acids (PUFAs). In the roots, $\omega-3$ fatty acid desaturase noticeably increased at low temperatures, whereas PUFA levels decreased.
\end{abstract}


Interestingly, C18:3 $3^{\Delta 9,12,15}$ alcohol was specifically found in safflower roots, and showed a significant increase, indicating a flux in the acid to alcohol ratio of this compound in safflower roots.

Key words: Safflower; Polyunsaturated fatty acids (PUFAs); Fatty acid desaturase; Real-time PCR; Gene expression

\section{INTRODUCTION}

The major fractions of polyunsaturated fatty acids (PUFAs) in plants are linoleic acid (LA, 18:2 $2^{\Delta 9,12}$ ) and a-linolenic acid (ALA, 18:3 $3^{\Delta 9,12,15}$ ). These 2 compounds are important structural components of membrane lipids and storage lipids in eukaryotic cells (Browse and Somerville, 1991). In addition to their important physiological role, LA and ALA are also essential for human health and nutrition, because they cannot be synthesized in the body and so must be obtained through the diet. In higher plants, PUFAs are synthesized via both prokaryotic (chloroplast) and eukaryotic (ER) pathways by a group of fatty acid desaturases (Roughan et al., 1980; Browse et al., 1986). The membrane-bound $\omega-6$ desaturase (codified by the microsomal FAD2 and the plastidial FAD6 genes) inserts a double bond between carbons 12 and 13 of oleic acid (18:1) to generate di-unsaturated linoleic acid (18:2) (Kargiotidou et al., 2008; Teixeira et al., 2009). In addition, $\omega-3$ desaturase (codified by 1 microsomal FAD3 and 2 plastidial $F A D 7$, and $F A D 8$ genes) further catalyzes the introduction of a 3rd bond between carbons 15 and 16 to form tri-unsaturated a-linolenic acid (18:3) (Niu et al., 2008; Teixeira et al., 2010).

Genes encoding both microsomal and platidial $\omega-3$ fatty acid desaturases have been isolated and studied from several plants species. Three independent microsomal $\omega-3$ desaturase genes, named GmFAD3A, GmFAD3B, and GmFAD3C, have been shown to contribute to soybean seed linolenic acid levels (Bilyeu et al., 2003). Two FAD3 desaturase genes were identified in flax (LuFAD3A and LuFAD3B) (Vrinten et al., 2005) and periila (PfFAD3A and PfFAD3B), which control the level of linolenic acid in seeds (Kim et al., 2008). Genes encoding plastidial $\omega-3$ desaturases (FAD7/8) were isolated from Arabidopsis thaliana (Iba et al., 1993; Gibson et al., 1994), Glycine max (van de Loo and Somerville, 1994), Zea mays (Berberich et al., 1998), and Descurainia sophia (Tang et al., 2007). Regulation of these genes has been shown to constitute a successful strategy for modifying the PUFA content of plant lipids.

Cultivated safflower (Carthamus tinctorius L.) has been traditionally characterized by high linoleic acid (18:2) content in the seed oil, representing more than 70\% of total fatty acid (FA), whereas linolenic acid (LNA, 18:3) was absent (Knowles, 1989; Velasco and FernandezMartinez, 2001). A similar observation was obtained for another Compositae family plant species, sunflower, which did not contain any LNA in seed storage oil; in contrast, the rest of the plant tissues contained relatively high levels of LNA fatty acids (Cantisán et al., 1999; Venegas-Calerón et al., 2006). Although the lipid content of safflower seed oil and its commercial value has been well documented, information about the regulatory mechanism of lipid biosynthesis in safflower seeds remains limited.

In this study, we isolated three $\omega-3$ desaturase genes (CtFAD3, CtFAD7, and CtFAD8) from safflower. The patterns of expression of these genes were investigated in different tis- 
sues. The relationships between the transcription level of $\omega-3$ desaturase genes and fatty acid composition were also investigated.

\section{MATERIAL AND METHODS}

\section{Plant materials}

Safflower (Carthamus tinctorius L.) Cultivar Chuan Hong 1 (12.5/80.2, \% oleic/linoleic), which was provided by Ms. Tang Li from Yaan San Jiu Medicine Co. Ltd, was grown in growth chamber. Young expanding leaves, roots, petioles, and young stems were harvested from 3-month-old seedlings of the cultivar, and were grown at $28^{\circ} \mathrm{C}$ and for cold-stress at $5{ }^{\circ} \mathrm{C}$ for 5 days, respectively. Flowers and seeds at different developmental stages (0 DAF, 5 DAF, $10 \mathrm{DAF}$, and $15 \mathrm{DAF}$ ) were harvested from self-pollinated safflower plants. Samples were stored at $-80^{\circ} \mathrm{C}$ until RNA extraction.

\section{RNA isolation and $R T$ reactions}

Total RNA was isolated from different safflower tissues using the Plant RNA Extraction kit (Tiangen, Beijing, China). The RNA concentration was determined spectrophotometrically, and verified by ethidium bromide staining on agarose gel. Total RNA was then treated with RNase-free DNase I (TaKaRa BIO INC, Dalian, China) to remove contaminating genomic DNA. Then, about $3 \mu \mathrm{g}$ was used as a template for the first cDNA synthesis using TaKaRa reverse transcription reagents, following the manufacturer protocols, and was stored at $-20^{\circ} \mathrm{C}$.

\section{Isolation of safflower $\omega$-3 desaturases partial cDNA clones}

The extracted cDNA was used as a template, in addition to PF1/PR1 and PF2/PR2 primers (Table 1) designed from $F A D 7 / 8$, and $F A D 3$ conserved sequences from other plants. PCR reactions were performed as follows: $95^{\circ} \mathrm{C}$ for $5 \mathrm{~min}$, followed by 35 cycles at $94^{\circ} \mathrm{C}$ for $30 \mathrm{~s}, 42^{\circ} \mathrm{C}$ for $30 \mathrm{~s}$, and $72^{\circ} \mathrm{C}$ for $1 \mathrm{~min}$, and finally $72^{\circ} \mathrm{C}$ for $7 \mathrm{~min}$. Different cDNA fragments of the same expected size were generated. All PCR fragments were subcloned into the vector pMD19-T (Promega), and transformed into E. coli JM109. Subsequently, a nucleotide sequence was determined by Invitrogen (Shanghai, China).

\section{Rapid amplification of cDNA ends (RACE)}

Nucleotide sequences of the $5^{\prime}$ end of the $\omega-3$ desaturase cDNA were amplified by the method of SMART rapid amplification of cDNA ends (RACE) 5'-RACE System (BDClontech, Palo Alto, CA, USA), following the manufacturer protocols. Two different antisense primers (Table 1) deduced from the 5'-region specific were used for each of the stated clones. Amplification of the $3^{\prime}$-end was performed by the $3^{\prime}$-RACE method, as described by Innis et al. (1990). The primers used for 3'-RACE were oligo (dT) 17 adaptor primer (for first cDNA synthesis) and 2 forward primers for each fragment (Table 1), following the manufacturer protocols. Based on the sequence information of the $5^{\prime}$ and $3^{\prime}$ end, the sense/antisense primer pairs (Table 1) were designed outside of the ORF, to amplify the full-length of the stated cDNAs. 


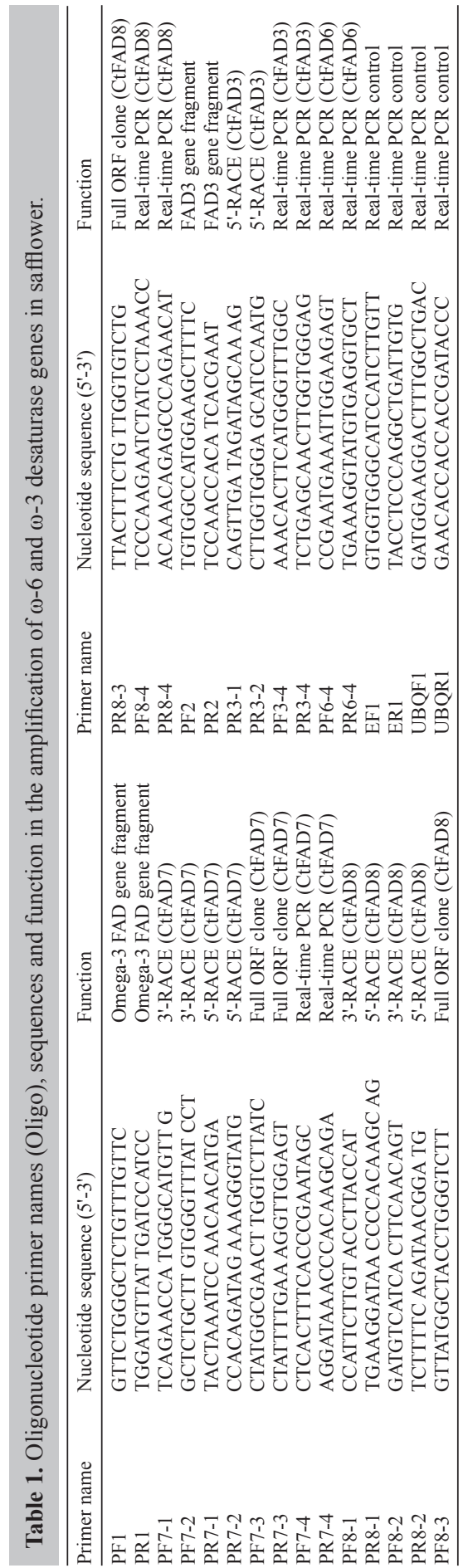




\section{Transcript analysis of $\omega$-3 desaturase gene by real-time reverse transcription PCR}

Specific primers (Table 1) for each gene were designed to amplify about $200 \mathrm{bp} \mathrm{PCR}$ products. As a control, parts of the coding region of the safflower ubiquitine 2 gene (CtUBQ2) and translation elongation factor $1 \mathrm{a}(C t E F-1 \mathrm{a})$ were amplified with the specific primers UBQF1 and UBQR1, and EF1 and ER1 (Table 1).

Quantitative PCR (Q-PCR) was carried out using the SYBR green I master mix (Tiangen, Beijing, China), with 3 replicates. Each $20 \mu \mathrm{L}$ reaction contained $9 \mu \mathrm{L}$ SYBR green master mix, $50 \mathrm{ng} \mathrm{cDNA}$, and $100 \mathrm{nM}$ forward and reverse primers. After a first denaturation step for $90 \mathrm{~s}$ at $94^{\circ} \mathrm{C}, 35$ cycles followed with $10 \mathrm{~s}$ at $94^{\circ} \mathrm{C}, 5 \mathrm{~s}$ at $55^{\circ} \mathrm{C}$, and $10 \mathrm{~s}$ at $72^{\circ} \mathrm{C}$. Fluorescence was measured 3 times at the end of the extension step at $72^{\circ}, 81^{\circ}$, and $84^{\circ} \mathrm{C}$. The identities of the amplicons and the specificity of PCR reactions were verified by agarose gel electrophoresis and melting curve analyses. PCR efficiencies (E) of all primers were calculated using dilution curves with 8 dilution points, 2 -fold dilution, and the equation: $\mathrm{E}=\left[10^{(-1 / \operatorname{slope})}\right]-1$. The housekeeping genes $C t U B Q 2$ and CtEF-1a (Czechowski et al., 2005; Reid et al., 2006) were used to normalize the gene mRNA level as an endogenous reference. The real-time PCR data were calibrated relative to the corresponding gene expression level following the $2^{-\Delta \Delta \mathrm{Ct}}$ method for relative quantification (Livak and Schmittgen, 2001). The data are presented as means \pm SD of 3 replications.

\section{Fatty acid analyses}

The fatty acid composition of safflower tissues was determined using the one-step method of Garces and Mancha (1993). Following the addition of $13.2 \mathrm{~mL}$ methanol/toluene/ dimethoxypropane $/ \mathrm{H}_{2} \mathrm{SO}_{4}(39: 20: 5: 2, \mathrm{v} / \mathrm{v} / \mathrm{v} / \mathrm{v})$ and $6.8 \mathrm{~mL}$ heptane to $300 \mathrm{mg}$ safflower tissue, the mixture was incubated for $1 \mathrm{~h}$ at $80^{\circ} \mathrm{C}$, forming a single phase. After cooling, the upper phase containing the fatty acid methyl esters was analyzed by the GC/MS-system using an Agilent 5973 MS system coupled with an Agilent 6890 gas chromatograph, fitted with an HP-SMS capillary column ( $30 \times 0.25 \mathrm{~mm}$, film thickness $0.25 \mu \mathrm{m}$ ). Helium was used as the carrier gas at $1 \mathrm{ml} / \mathrm{min}$, the injector temperature was $250^{\circ} \mathrm{C}$, the split ratio was $50: 1$, and the temperature program was set to increase by $4^{\circ} \mathrm{C}$ every minute from $120^{\circ}$ to $220^{\circ} \mathrm{C}$.

\section{Sequence analyses}

Three nucleotide sequences of our cDNA clones and the deduced amino acid (aa) sequences (designed CtFAD3, CtFAD7, and CtFAD8) were identified by the NCBI BLAST program (http://www.ncbi.nlm.nih.gov/BLAST/), and have been submitted to the GenBank database. The assigned accession numbers were HQ831356 (CtFAD3), HQ831349 (CtFAD7), and HQ831350 (CtFAD8). All of the other sequences used for phylogenetic analysis were retrieved from the GenBank and are listed in Table 2. Sequences were selected with the aim to cover most of the living world and to avoid "redundancy" for each desaturase. Analysis of the nucleotide sequences and protein alignment was performed by DNAMAN software (version 4.0, Lynnon Biosoft, Quebec). Phylogenetic trees were generated by the neighbor-joining method, implemented in the MEGA 4.0 software (Tamura et al., 2007) with 500 bootstraps. The prediction of a chloroplast transit peptide was performed by the online program ChloroP 1.1 server (http:// www.cbs.dtu.dk/services/ChloroP/). Transmembrane regions were predicted by the TMHMM 
server v. 2.0 (http://www.cbs.dtu.dk/ services/TMHMM/). Predictions of subcellular localization of the deduced polypeptides were conducted by PSORT (http://psort.hgc.jp/form.html).

\begin{tabular}{|c|c|c|c|c|c|}
\hline \multirow[t]{2}{*}{ Categories } & \multirow[t]{2}{*}{ Scientific name (abbreviation) } & \multicolumn{2}{|c|}{ Microsomal $\omega-3$ fatty acid desaturas } & \multicolumn{2}{|c|}{ Plastidial $\omega-3$ fatty acid desaturas } \\
\hline & & Gene & Accession No. & Gene & Accession No. \\
\hline \multirow[t]{34}{*}{ Dicotyledon } & \multirow[t]{2}{*}{ Arabidopsis thaliana $(\mathrm{At})$} & FAD3 & d26508 & FAD7 & d14007 \\
\hline & & & & FAD8 & 127158 \\
\hline & \multirow[t]{2}{*}{ Brasicca napus $(\mathrm{Bn})$} & FAD3 & 122962 & FAD7 & fj985690 \\
\hline & & - & - & FAD8 & fj985691 \\
\hline & \multirow[t]{2}{*}{ Betula pendula $(\mathrm{Bp})$} & FAD3 & ay135566 & FAD7 & ay 135565 \\
\hline & & & & FAD8 & ay135564 \\
\hline & Crepis alpina $(\mathrm{Ca})$ & FAD3 & aba55806 & FAD7 & aba55807 \\
\hline & Cucumis sativus $(\mathrm{Cs})$ & - & - & FAD7 & eu723878 \\
\hline & \multirow{2}{*}{ Carthamus tinctorius $(\mathrm{Ct})$} & FAD3 & HQ831356 & FAD7 & HQ831349 \\
\hline & & - & - & FAD8 & HQ831350 \\
\hline & \multirow[t]{2}{*}{ Descurainia sophia $(\mathrm{Ds})$} & FAD3 & ef105162 & FAD7 & ef105163 \\
\hline & & - & - & FAD8 & ef105164 \\
\hline & \multirow[t]{3}{*}{ Glycine $\max (\mathrm{Gm})$} & FAD3A & ay204710 & FAD7-1 & gq144962 \\
\hline & & FAD3B & ef632325 & FAD7-2 & eu621390 \\
\hline & & FAD3C & ef632326 & FAD8 & fj393229 \\
\hline & Helianthus annuus $(\mathrm{Ha})$ & - & - & FAD7 & ay 254858 \\
\hline & \multirow[t]{2}{*}{ Jatropha curcas $(\mathrm{Jc})$} & FAD3 & abx82798 & FAD7 & abe 72960 \\
\hline & & - & - & FAD8 & abu96743 \\
\hline & \multirow[t]{2}{*}{ Olea europaea $(\mathrm{Oe})$} & FAD3 & abg88130 & FAD7 & abg88131 \\
\hline & & & - & FAD8 & dq788674 \\
\hline & \multirow[t]{2}{*}{ Linum usitatissimum $(\mathrm{Lu})$} & FAD3A & dq116424 & - & - \\
\hline & & FAD3B & dq116425 & - & - \\
\hline & \multirow{3}{*}{ Perilla frutescens $(\mathrm{Pf})$} & FAD3 & d26509 & FAD7 & ab049577 \\
\hline & & FAD3A & af 213482 & FAD7 & u59477 \\
\hline & & FAD3B & af047039 & & - \\
\hline & Prunus persica $(\mathrm{Pp})$ & - & - & FAD7 & aam77643 \\
\hline & Ricinus communis $(\mathrm{Rc})$ & FAD3 & eef36775 & FAD7 & 125897 \\
\hline & Sesamum indicum $(\mathrm{Si})$ & - & - & FAD7 & u25817 \\
\hline & \multirow[t]{2}{*}{ Solanum lycopersicum $(\mathrm{Sl})$} & FAD3 & abx 24525 & FAD7 & aap82170 \\
\hline & & - & - & FAD8 & aan62759 \\
\hline & Solanum tuberosum $(\mathrm{St})$ & - & - & FAD7 & caa07638 \\
\hline & Triadica sebifera $(\mathrm{Ts})$ & FAD3 & ef044310 & - & - \\
\hline & \multirow[t]{2}{*}{ Vernonia galamensis $(\mathrm{Vg})$} & FAD3 & eu180594 & FAD7 & eu180596 \\
\hline & & - & - & FAD8 & eu180595 \\
\hline \multirow[t]{5}{*}{ Monocotyledons } & \multirow[t]{2}{*}{ Oryza sativa $(\mathrm{Os})$} & FAD3 & d78505 & FAD7 & ab232382 \\
\hline & & - & & FAD8 & ab232383 \\
\hline & \multirow{3}{*}{$\begin{array}{l}\text { Triticum aestivum }(\mathrm{Ta}) \\
\text { Zea mays }(\mathrm{Zm})\end{array}$} & FAD3 & d84678 & FAD7 & d443688 \\
\hline & & - & - & FAD7 & $\mathrm{d} 63954$ \\
\hline & & - & - & FAD8 & D84409 \\
\hline \multirow[t]{5}{*}{ Prokaryota } & Cyanothece sp (csp) & FAD3 & zp01728541 & - & - \\
\hline & Synechococcus sp (ssp) & FAD3 & aab61352 & - & - \\
\hline & Nodularia $\mathrm{sp}(\mathrm{N} \mathrm{sp})$ & FAD3 & np485637 & - & - \\
\hline & Oscillatoria sp (Osp) & FAD3 & zp07111339 & - & - \\
\hline & Lyngbya sp (Lsp) & FAD3 & zр01624560 & - & - \\
\hline
\end{tabular}

List is ordered by species.

\section{RESULTS}

\section{Isolation and characterization of safflower $\omega$-3 desaturase genes}

Based on the nucleotide sequences of FAD3 and FAD7/8 from different plant species, specific primers were designed to amplify the partial cDNA fragments of the microsomal and plastidial $\omega-3$ desaturase genes. Five different fragments were obtained, with an expected size of 
about $900 \mathrm{bp}$. Alignment of the deduced aa sequences of these fragments showed a high degree of identity to the central coding regions of known plant $\omega-3$ desaturase gene sequences. Therefore, they were designated as CtFAD3,CtFAD7, and CtFAD8. To obtain full-length cDNA clones, we performed 3'- and 5'-RACE, using gene-specific primers designed from the known cDNA fragments. Sequence comparisons of the 5'- and 3'- ends with the conservative parts of the CtFAD7 and CtFAD 8 genes indicated a perfect match for the overlapping regions, with sizes 1675 and $1680 \mathrm{bp}$. These sequeces revealed ORFs encoding predicted proteins of 441 and 440 amino acid (aa) residues, respectively, which correspond to a calculated molecular mass of 50.40 and 50.25 $\mathrm{kDa}$. With respect to CtFAD3, the 5'-end sequence matched the overlapping regions, but the 3'end did not match. Therefore, we did not obtain the $3^{\prime}$ region of $C t F A D 3$ in this study.

Multiple sequence alignment revealed that CtFAD3, CtFAD7, and CtFAD 8 showed high similarities (60-93\%, 61-79\%, and 63-78\%, respectively) with other orthologous desaturases in the GenBank. The aa sequence identity between CtFAD7 and CtFAD 8 was $82.5 \%$, while both showed much lower identity to CtFAD3 (64.2\% and 64.8\%, respectively). All of the safflower $\omega-3$ desaturases contain 3 histidine centers (HXXXH, HXXHH, and HXXHH) typical of plant $\omega-3$ desaturases (Los and Murata, 1998). Analysis with the TargetP Server (Emanuelsson et al., 2000) found putative chloroplast transit peptides, 56 and 27 aa, at the Ntermini of CtFAD7 and CtFAD8 (Figure 1).

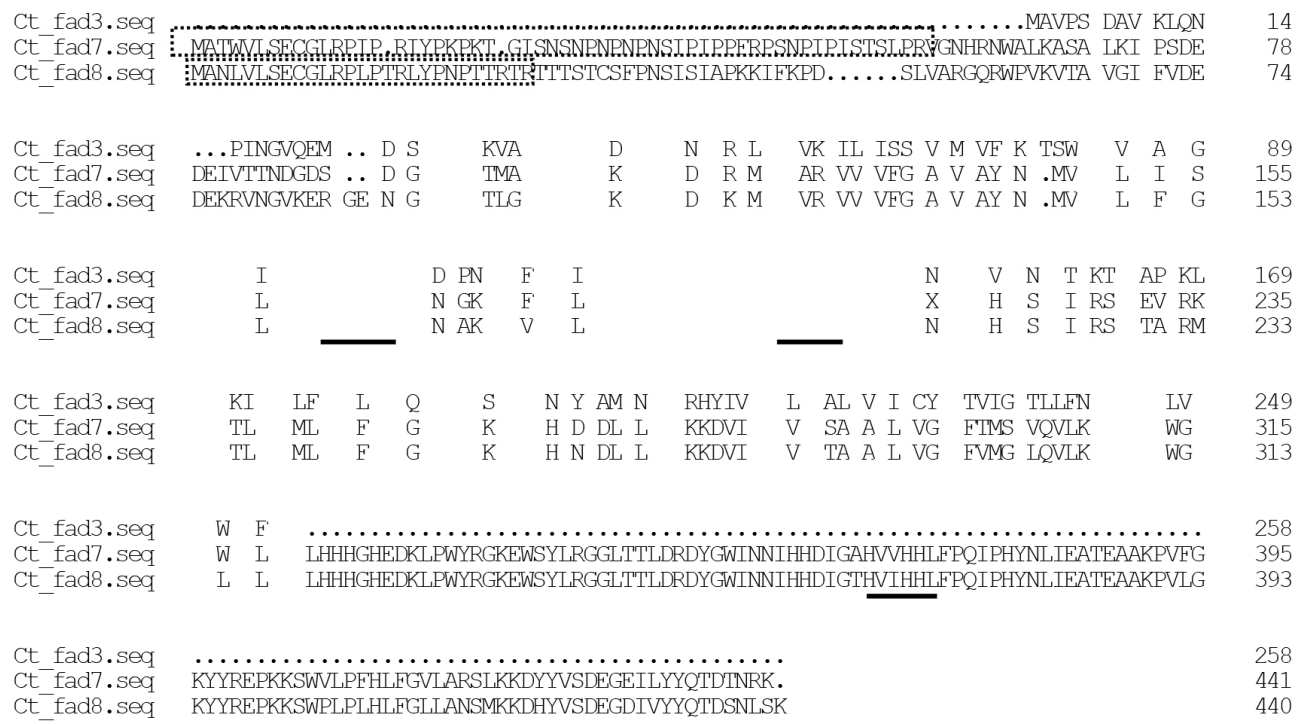

Figure 1. Alignment of the deduced aa sequences determined for safflower $\omega-3$ fatty acid desaturases. Identical or similar aa are shaded black or blue, respectively. The three characteristic histidine boxes are underlined. N-terminal signal peptide of FAD7/8 are represented by boxes.

\section{Phylogenetic relationships of plant $\omega-3$ desaturase genes}

Using safflower fatty acid desaturases and other plant $\omega-3$ desaturase sequences from the National Center for Biotechnology Information (NCBI) (Table 2), we generated a phylogenetic tree (Figure 2). The neighbor-joining phylogenetic analysis revealed 3 distinct clusters within the 
$\omega$-3 desaturases: (1) a group integrated by the $\omega-3$ desaturases from cyanobacteria, (2) a group with the microsomal $\omega-3$ desaturases from plants, and (3) a group formed by the chloroplast $\omega-3$ desaturases from plant. The species distribution in the last 2 clusters was essentially consistent with conventional species trees, separating Monocotyledonae from Dicotyledonae species. This result also indicated that the origin of $\omega-3$ desaturases took place in the prokaryotic lineage, before the appearance of plants. Phylogenetic relationships of CtFAD7 with CtFAD8 were closer, both of them located in the plant plastidial $\omega-3$ desaturase group. In comparison, CtFAD3 was grouped in the branch of Dicotyledonae microsomal $\omega-3$ desaturases.

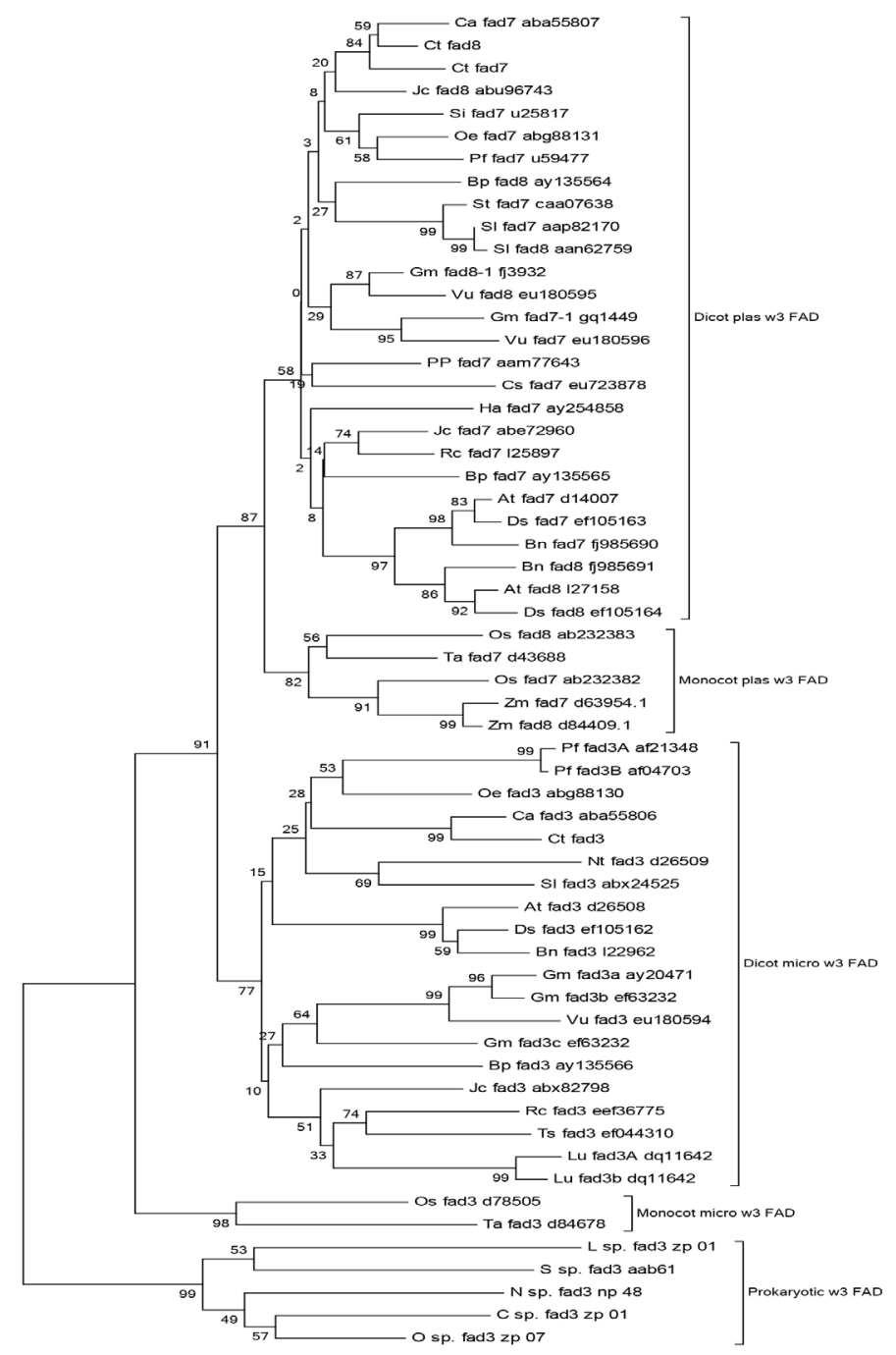

Figure 2. Phylogenetic relationships of $\omega-3$ desaturases from different plants. Position of the safflower $\omega-3$ desaturases were underlined. The protein sequences were obtained from GenBank, and the accession numbers were listed in Table 2 . The tree was constructed by the neighbor-joining algorithm. Numbers on the nodes indicate bootstrap values after 500 replicates. Plant stearic ACP enzymes were used as outgroup. 


\section{Fatty acid composition and the transcriptional levels of $\omega-3$ desaturase genes in different safflower plant tissues}

Our results showed that both $C t F A D 7$ and $C t F A D 8$ genes were expressed in vegetative tissues, with the highest expression level in leaves, and with extremely low levels in the flowers and seeds. As shown in Figure 3B, CtFAD3 mRNA seemed to be constitutively expressed in all vegetative tissues, showing higher transcriptional levels in mature leaves and flowers. In non-photosynthetic tissues, such as the roots, stems, and flowers, CtFAD3 was the major $\omega-3$ desaturase gene detected, indicating that $C t F A D 3$ plays an important role in linolenic acid syntheses in these tissues. Different from all of the other oil crop microsomal $\omega-3$ desaturases, the transcription level of CtFAD3 in safflower developing seeds was extremely low (Figure 3A, B).
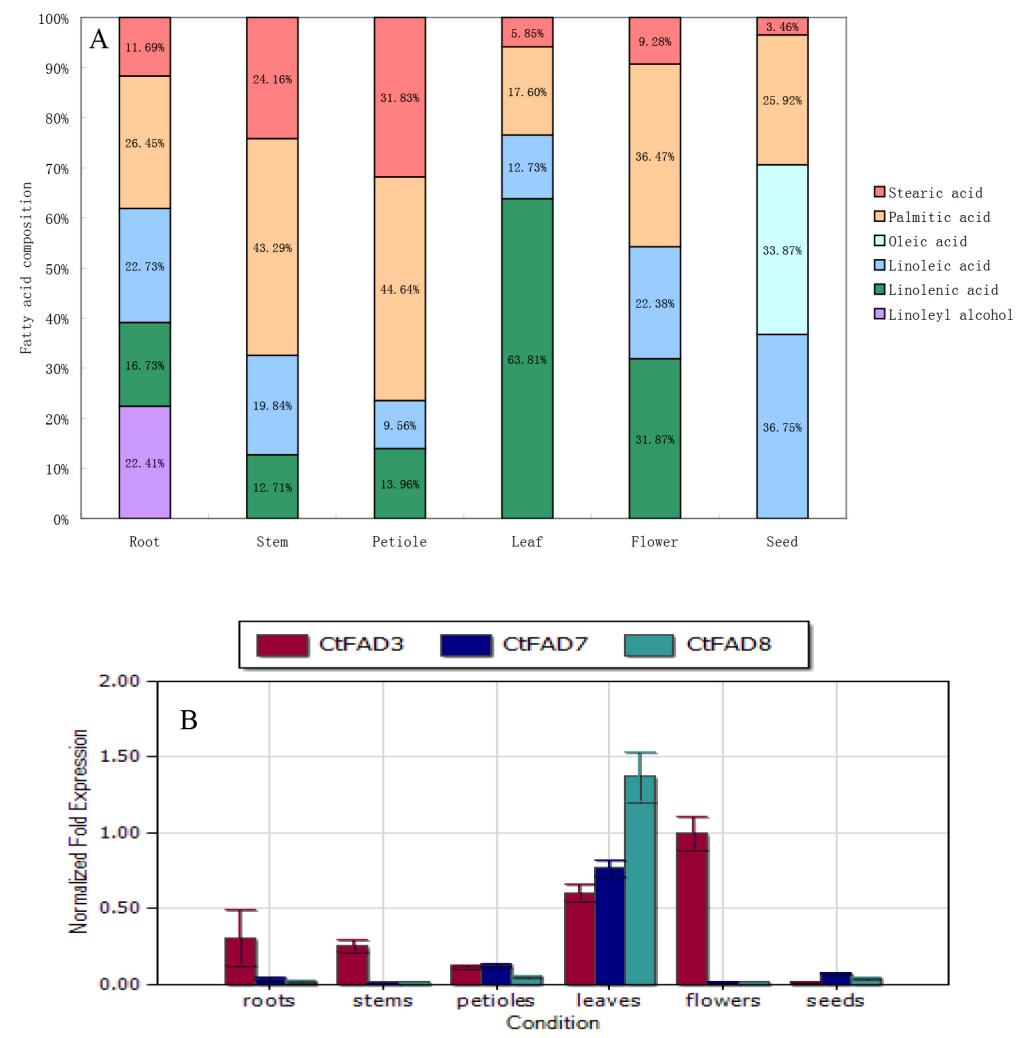

Figure 3. Fatty acid composition (A) and relative expression levels of safflower $\omega-3$ desaturase genes (B). Fatty acid composition and relative expression levels were determined in the indicated tissues as described in Material and Methods.

The fatty acid composition of safflower seeds has been previously analyzed by many research groups. However, the characteristics of fatty acid composition in vegetative tissues have not been analyzed in detail, until this study. As shown in Figure 3A, safflower vegetative tissues contained 2 main PUFAs, LNA (18:3) and LA (18:2), and 2 types of saturated fatty acids, palmitic acid (PAL, 16:0) and stearic acid (STE, 18:0). Safflower belongs to the 
group of so-called "18:3 [LNA] plants," i.e., it does not contain 16:3 in its cell membranes (Roughan et al., 1980). One difference between safflower and other LNA plant species was that 18:1 was not present in these tissues. The highest amount of trienoic fatty acids (i.e., LNA, 18:3) was observed in the leaves, representing more than $60 \%$ of total fatty acids, which was similar to other LNA plants (Andreu et al., 2010). In flowers, the LNA level was more than $30 \%$, while LNA was not detected at all in developing seeds. These results were consistent with the expression patterns of $\omega-3$ genes in these tissues, indicating the direct positive correlations between the transcriptional levels of $\omega-3$ genes and LNA accumulations. In the roots, the contents of LA and LNA were relatively low. Unexpectedly, high percentages of another $\mathrm{C} 18: 3^{\Delta 9,12,15}$ component, $\mathrm{C} 18: 3^{\Delta 9,12,15}$ alcohol, were detected in the safflower roots (more than $20 \%$ ). The gas chromatography analysis of fatty acid (FA) composition and mass spectral identification of $\mathrm{C} 18: 3^{\Delta 9,12,15}$ alcohol are shown in Figure 4.

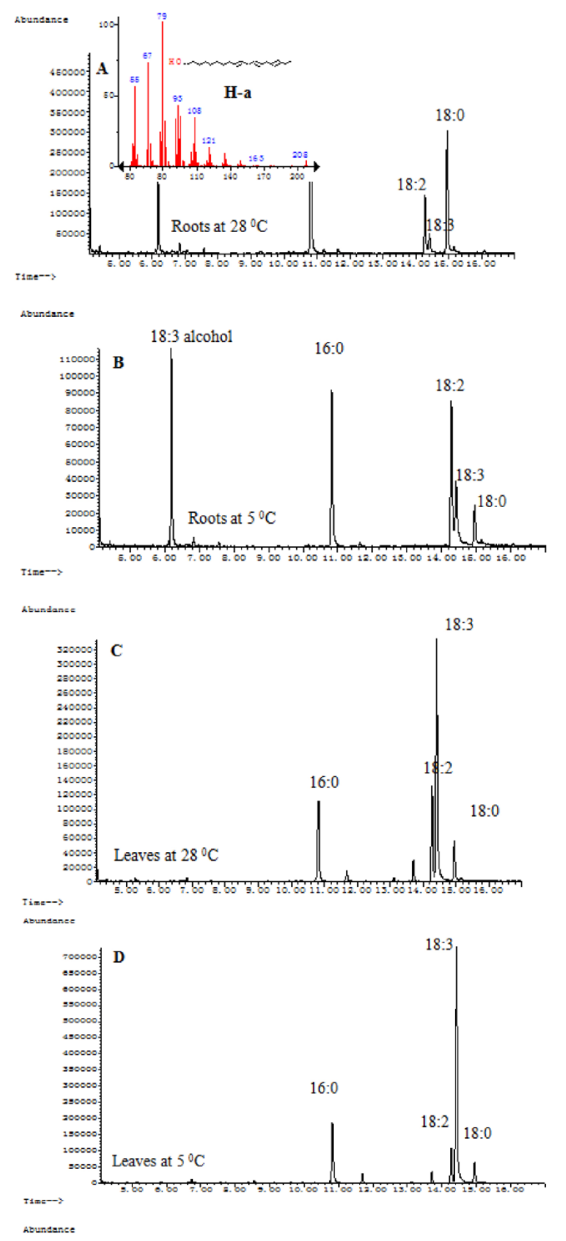

Figure 4. Representative fatty acid methyl ester profiles of safflower vegetative tissues at $28^{\circ}$ and $5^{\circ} \mathrm{C}$. A. B. fatty acids in roots. $\mathrm{H}-\mathrm{a}=$ Mass spectral identification of $\mathrm{C} 18: 3^{\Delta 9,12,15}$ alcohol from safflower roots. C. D. Fatty acids in leaves at $5^{\circ}$ and $28^{\circ} \mathrm{C}$. 


\section{Impact of low temperature on the transcriptional levels of $\omega-3$ desaturase genes and the accumulation of fatty acids in vegetative tissues}

The unsaturated fatty acid (PUFAs) levels in plant vegetative tissues might be affected by growth temperature (Kinney, 1998). To test whether this temperature effect is caused by enhanced gene expression, the fatty acid composition and the expression levels of $\omega-3$ desaturase genes were determined in safflower vegetative tissues cultivated at $28^{\circ} \mathrm{C}$ and $5^{\circ} \mathrm{C}$ (Figure $5 \mathrm{~A}, \mathrm{~B}, \mathrm{C}$ and D). Our results showed that the percentages of LNA in leaves slightly increased from 63.31 to $67.27 \%$, but at the expense of LA, which decreased from 12.73 to $8.70 \%$. Both LA and LNA increased in the stems and petioles, but were accompanied with a decrease in 2 saturated fatty acids (16:0 and 18:0). Interestingly, the percentages of both LA and LNA decreased in the roots after cold inducement, whereas C18:3 $3^{\Delta 9,12,15}$ alcohol significantly increased from 22.41 to $32.13 \%$ (Figure $5 \mathrm{~A}$ ).

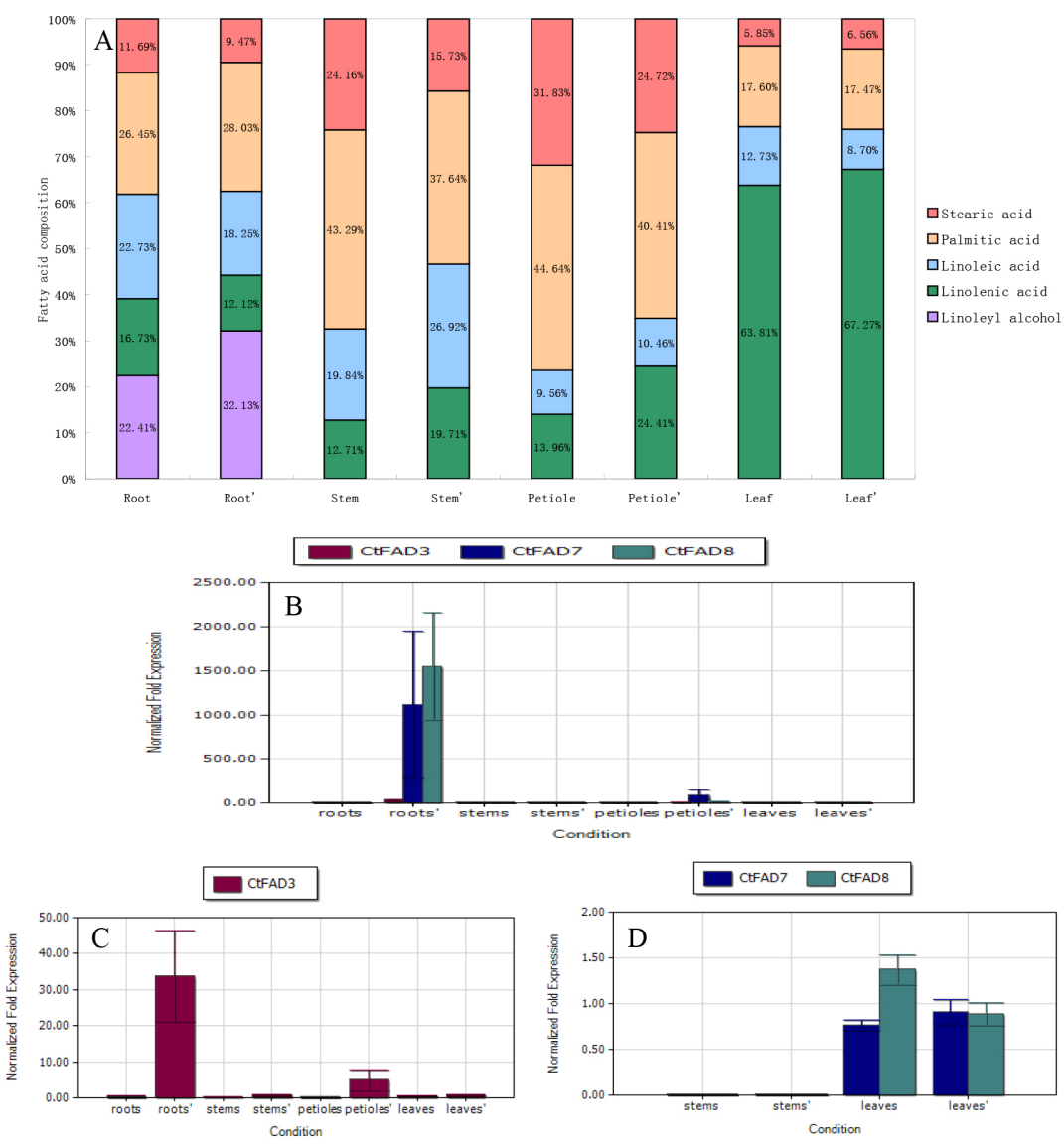

Figure 5. Growth temperature effects on fatty acid compositions and the expression of $\omega-6$ and $\omega-3$ desaturase genes in safflower vegetative tissues. A. Fatty acids composition at $28^{\circ}$ and $5^{\circ} \mathrm{C}$. B. Total information on the transcript levels of the safflower $\omega-3$ desaturase genes at different temperature. C. Temperature effects on transcript levels of CtFAD3 in different tissues. D. Temperature effects on transcript levels of CtFAD7 and CtFAD 8 in stems and leaves. 
The expressions of $\omega-3$ desaturase genes at different temperatures are shown in Figure 5B. In the leaves, the transcription level of $C t F A D 3$ remained constant at all different growth temperatures; in contrast, the accumulation of CtFAD7 mRNA slightly increased at low temperature, while CtFAD 8 mRNA decreased significantly. In the stems, the transcription levels of the three $\omega-3$ desaturase genes were not affected by low temperature, indicating that the elevated LNA levels in the stems at low temperatures were not caused by the enhanced expression of $\omega-3$ desaturase genes. In the petioles, $C t F A D 3$ and $C t F A D 7$ slightly increased, which coincided with the increased LNA percentages. The expressions of CtFAD3, CtFAD7, and CtFAD 8 in the roots significantly increased at low temperature. This result seems contradictory to the decreased percentages of LNA. In addition, the level of C18:3 $3^{\Delta 9,12,15}$ alcohol significantly increased from 22.41 to $32.13 \%$.

\section{DISCUSSION}

This study described the identification of $\omega$-3 desaturases genes from safflower. Sequence analysis showed that these safflower $\omega-3$ desaturases shared all of the conserved features, including hydrophobic residues, which might traverse the lipid bilayer (McCartney et al., 2004) and three histidine boxes that essential for enzyme activity (Los and Murata, 1998).

Safflower seed oil has been analyzed by many research groups (Futehally and Knowles, 1981; Bergman et al., 2006); however, information about the characters of fatty acid composition in safflower vegetative tissue has not been reported before. In this study, the analysis of FA composition in different vegetative tissues of safflower showed that safflower belongs to the group of so-called "18:3 plants," (LNA) i.e., it does not contain 16:3 in its cell membranes (Roughan and Slack, 1982; Monteiro et al., 1990; Mongrand et al., 1998). In contrast to all existing reports about FA composition in plant species, there was no 18:1 present in safflower vegetative tissues. We assumed that this phenomenon was associated with the large copy numbers of $\omega-6$ desaturase genes present in safflower, which was much greater compared to any other plant species (Guan, 2012a,b; Cao et al., 2013). In addition, stearic-ACP desaturase might be the restricted enzyme in the process of PUFA analysis in safflower vegetative tissues.

Unlike other oilseed plants, LNA is not present in the seed oil of safflower and sunflower. In contrast, the rest of the plant tissues contain relatively high levels of LNA fatty acid (Cantisán et al., 1999; Venegas-Calerón et al., 2006). In sunflower, only one $\omega$-3 desaturase gene, HaFAD7, was isolated in the contribution of LNA synthesis in sunflower vegetative tissues (Venegas-Calerón et al., 2006). In this study, we isolated 2 plastidial $\omega-3$ desaturases, CtFAD7 and CtFAD8, and 1 microsomal $\omega-3$ desaturase (CtFAD3) partial cDNA. In oil crops, FAD3 desaturase is an essential enzyme in the production of LNA during seed development. The regulation of the FAD3 gene has proven a successful strategy for modifying the LNA properties of seed oils (Bilyeu et al., 2003; Vrinten et al., 2005; Kim et al., 2008). The presence of FAD3 in safflower raised the question of why it does not contribute LNA synthesis in safflower seeds. We did not obtain the full length cDNA of CtFAD3; therefore, we have no direct evidence of whether the low level of LNA in safflower seeds is caused by the loss of FAD3 enzyme activity or by promoter mediation. However, in our study, we did determine the fatty acid composition and the transcription level of FAD 3 in vegetative tissues and developing seeds (Figure $3 \mathrm{AB}$ ). The high level of LNA content and CtFAD3 transcription in the flowers, along with the extremely low level of $C t F A D 7$ and $C t F A D 8$, indicated that $C t F A D 3$ plays the leading role in the synthesis 
of LNA in flower tissue. This finding might provide indirect evidence for the full enzyme activity of $C t F A D 3$, indicating that the low level of LNA in safflower seeds was not caused by the loss of CtFAD3 enzyme activity. Therefore, we investigated the transcription level of CtFAD3 in developing seeds. Our results showed that the transcription of CtFAD3 in safflower seeds was extremely low. This finding indicated that the absence of LNA in safflower seeds was caused by the regulation of $C t F A D 3$ transcription, rather than gene mutation.

Except for safflower seeds, the FA composition of vegetative tissue was highly influenced by growth temperature. Comparison of the transcription of $\omega-3$ desaturases with changes in fatty acids indicated the presence of regulation at both the transcription and post-transcription level. In the stems and petioles, the increased transcription levels of $\omega-3$ desaturase genes resulted in increased LNA. Of interest, the significantly increased $\omega-3$ desaturase genes did not cause an increase in PUFAs in the safflower roots; yet, both LA and LNA noticeably decreased. In addition, we detected another $\mathrm{C} 18: 3$ composition, $\mathrm{C} 18: 3^{\Delta 9,12,15}$ alcohol, which was primarily present in safflower roots (Figure 4). The C18:3 ${ }^{\Delta 9,12,15}$ alcohol significantly increased when the plant was grown at low temperature. Based on the increased transcription level of $\omega-3$ desaturase genes, we assumed that there is a flux in $\mathrm{C} 18: 3^{\Delta 9,12,15}$ acid to $\mathrm{C} 18: 3^{\Delta 9,12,15}$ alcohol, which causes a decrease in LA and LNA. The theory of "homeoviscous acclimation" (also known as increasing the level of membrane PUFAs) states that the maintaining the appropriate fluidity of membrane lipids protects against ambient temperature changes (Mongrand et al., 1998). This theory might provide one possible explanation for the unexpected observation of this study, causing the conversion of $18: 3$ acid to $C 18: 3^{\Delta 9,12,15}$ alcohol. This conversion might benefit the safflower plant by allowing it to maintain membrane fluidity in roots to cope with cold stress, because of the better fluidity of alcohol compared to fatty acids in colder temperatures.

\section{ACKNOWLEDGMENTS}

Research supported by the National Natural Science Foundation of China (\#181274020 and \#81202910).

\section{REFERENCES}

Andreu V, Lagunas B, Collados R, Picorel R, et al. (2010). The GmFAD7 gene family from soybean: identification of novel genes and tissue-specific conformations of the FAD7 enzyme involved in desaturase activity. J. Exp. Bot. 61: 3371-3384.

Berberich T, Harada M, Sugawara K, Kodama H, et al. (1998). Two maize genes encoding omega-3 fatty acid desaturase and their differential expression to temperature. Plant Mol. Biol. 36: 297-306.

Bergman JW, Riveland NR, Flynn CR, Carlson GR, et al. (2006). Registration of "Montola 2004" safflower. Crop Sci. 46: 1818-1819.

Bilyeu KD, Palavalli L, Sleper DA and Beuselinck PR (2003). Three microsomal omega-3 fatty-acid desaturase genes contribute to soybean linolenic acid levels. Crop Sci. 43: 1833-1838.

Browse J, Warwick N, Somerville CR and Slack CR (1986). Fluxes through the prokaryotic and eukaryotic pathways of lipid synthesis in the '16:3' plant Arabidopsis thaliana. Biochem. J. 235: 25-31.

Browse J and Somerville C (1991). Glycerolipid synthesis: biochemistry and regulation. Annu. Rev. Plant Physiol. Plant Mol. Biol. 42: 456-506.

Cantisán S, Martinez-Force E, Alvarez-Ortega R and Garces R (1999). Lipid characterization in vegetative tissues of high saturated fatty acid sunflower mutants. J. Agric. Food Chem. 47: 78-82.

Cao S, Zhou XR, Wood CC, Green AG, et al. (2013). A large and functionally diverse family of Fad2 genes in safflower 
(Carthamus tinctorius L.). BMC Plant Biol. 13: 5.

Czechowski T, Stitt M, Altmann T, Udvardi MK, et al. (2005). Genome-wide identification and testing of superior reference genes for transcript normalization in Arabidopsis. Plant Physiol. 139: 5-17.

Emanuelsson O, Nielsen H, Brunak S and von HG (2000). Predicting subcellular localization of proteins based on their N-terminal amino acid sequence. J. Mol. Biol. 300: 1005-1016.

Futehally S and Knowles PF (1981). Inheritance of Very High Levels of Linoleic Acid in an Introduction of Safflower (Carthamus tinctorius L.) from Portugal. In: First International Safflower Conference, Davis, 56-61.

Garces R and Mancha M (1993). One-step lipid extraction and fatty acid methyl esters preparation from fresh plant tissues. Anal. Biochem. 211: 139-143.

Gibson S, Arondel V, Iba K and Somerville C (1994). Cloning of a temperature-regulated gene encoding a chloroplast omega-3 desaturase from Arabidopsis thaliana. Plant Physiol. 106: 1615-1621.

Guan LL, Wang YB, Shen H, Hou K, et al. (2012a). Molecular cloning and expression analysis of genes encoding two microsomal oleate desaturases (FAD2) from safflower (Carthamus tinctorius L.). Plant mol. Boil. Rep. 30: 139-148.

Guan LL, Xu YW, Wang YB, Chen L, et al. (2012b). Isolation and characterization of temperature-regulated microsomal oleate desaturase gene (FAD2) from normal type and high oleic safflower (Carthamus tinctorius L.). Plant Mol. Biol. Rep. 30: 391-402.

Iba K, Gibson S, Nishiuchi T, Fuse T, et al. (1993). A gene encoding a chloroplast omega-3 fatty acid desaturase complements alterations in fatty acid desaturation and chloroplast copy number of the fad 7 mutant of Arabidopsis thaliana. J. Biol. Chem. 268: 24099-24105.

Innis MA, Gelfand DH, Sninsky JJ and White TJ (1990). PCR Protocols: A Guide to Method and Application. (Frohman MA, ed.). Academic Press, San Diego, 28-38.

Kargiotidou A, Deli D, Galanopoulou D, Tsaftaris A, et al. (2008). Low temperature and light regulate delta 12 fatty acid desaturases (FAD2) at a transcriptional level in cotton (Gossypium hirsutum). J. Exp. Bot. 59: 2043-2056.

Kim MJ, Go YS, Ahn SJ, Chung CH, et al. (2008). Functional complementation of a periila $\omega 3$ fatty acid desaturase under the seed-specific SeFAD2 promoter. J. Plant Biol. 51: 174-179.

Kinney AJ (1998). Manipulating flux through plant metabolic pathways. Curr. Opin. Plant Biol. 1: 173-178.

Knowles PF (1989). Safflower. In: Oil Crops of the World (Downey RK, Robbelen G and Ashri A, eds.). New York, 363-374.

Livak KJ and Schmittgen TD (2001). Analysis of relative gene expression data using real-time quantitative PCR and the $2^{-\Delta \Delta C t}$ Method. Methods 25: 402-408.

Los DA and Murata N (1998). Structure and expression of fatty acid desaturases. Biochim. Biophys Acta 1394: 3-15.

McCartney AW, Dyer JM, Dhanoa PK, Kim PK, et al. (2004). Membrane-bound fatty acid desaturases are inserted cotranslationally into the ER and contain different ER retrieval motifs at their carboxy termini. Plant J. 37: 156-173.

Mongrand S, Bessoule JJ, Cabantous F and Cassagne C (1998). The C16-3/C18-3 fatty acid balance in photosynthetic tissues from 468 plant species. Phytochemistry 49: 1049-1064.

Monteiro PF, Pham-Thi AT, Vieira SJ, Justin AM, et al. (1990). Effects of water stress on the molecular species composition of polar lipids from Vigna unguiculata L. leaves. Plant Sci. 66: 85-193.

Niu B, Guo L, Zhao M, Luo T, et al. (2008). Molecular cloning, characterization, and expression of an omega-3 fatty acid desaturase gene from Sapium sebiferum. J. Biosci. Bioeng. 106: 375-380.

Reid KE, Olsson N, Schlosser J, Peng F, et al. (2006). An optimized grapevine RNA isolation procedure and statistical determination of reference genes for real-time RT-PCR during berry development. BMC Plant Biol. 6: 27.

Roughan PG, Holland R and Slack CR (1980). The role of chloroplasts and microsomal fractions in polar-lipid synthesis from [1-14C] acetate by cell-free preparations from spinach (Spinacia oleracea) leaves. Biochem. J. 188: 17-24.

Roughan PG and Slack CR (1982). Cellular organization of glycerolipid metabolism. Ann. Rev. Plant Physiol. 33: 97-112.

Tamura K, Dudley J, Nei M and Kumar S (2007). MEGA4: Molecular Evolutionary Genetics Analysis (MEGA) software version 4.0. Mol. Biol. Evol. 24: 1596-1599.

Tang S, Guan R, Zhang H and Huang J (2007). Cloning and expression analysis of three cDNAs encoding omega-3 fatty acid desaturases from Descurainia sophia. Biotechnol. Lett. 29: 1417-1424.

Teixeira MC, Coelho N, Olsson ME, Brodelius PE, et al. (2009). Molecular cloning and expression analysis of three omega-6 desaturase genes from purslane (Portulaca oleracea L.). Biotechnol. Lett. 31: 1089-1101.

Teixeira MC, Carvalho IS and Brodelius M (2010). Omega-3 fatty acid desaturase genes isolated from purslane (Portulaca oleracea L.): expression in different tissues and response to cold and wound stress. J. Agric. Food Chem. 58: 18701877.

van de Loo FJ and Somerville C (1994). Plasmid omega-3 fatty acid desaturase cDNA from Ricinus communis. Plant Physiol. 105: 443-444.

Velasco L and Fernandez-Martinez JM (2001). Breeding for oil Quality in Safflower. In: Proceedings of the 5th

Genetics and Molecular Research 13 (3): 6623-6637 (2014)

CFUNPEC-RP www.funpecrp.com.br 
International Safflower Conference (Bergman JW and Mündel HH, eds.), Williston and Sidney, 133-137.

Venegas-Calerón M, Muro-Pastor AM, Garces R and Martinez-Force E (2006). Functional characterization of a plastidial omega-3 desaturase from sunflower (Helianthus annuus) in cyanobacteria. Plant Physiol. Biochem. 44: 517-525.

Vrinten P, Hu Z, Munchinsky MA, Rowland G, et al. (2005). Two FAD3 desaturase genes control the level of linolenic acid in flax seed. Plant Physiol. 139: 79-87. 